\title{
Modulational instability of solitary waves in non-degenerate three-wave mixing: The role of phase symmetries.
}

\author{
Dmitry V. Skryabin *, and William J. Firth \\ Department of Physics and Applied Physics, John Anderson Building, \\ University of Strathclyde, 107 Rottenrow, Glasgow, G4 0NG, Scotland
}

(April 8, 1998)

We show how the analytical approach of Zakharov and Rubenchik [Sov. Phys. JETP 38, 494 (1974)] to modulational instability (MI) of solitary waves in the nonlinear Schröedinger equation (NLS) can be generalised for models with two phase symmetries. MI of three-wave parametric spatial solitons due to group velocity dispersion (GVD) is investigated as a typical example of such models. We reveal a new branch of neck instability, which dominates the usual snake type MI found for normal GVD. The resultant nonlinear evolution is thereby qualitatively different from cases with only a single phase symmetry.

One of the central issues of solitary wave theory is the question of stability [1,2]. Stability of solitary waves ('solitons') in nonintegrable Hamiltonian models is often governed by the derivative of a certain invariant with respect to an associated parameter of the solution [1] 3 . For instance, positivity of the derivative of the total energy with respect to the nonlinearity-induced wavenumber shift is sufficient for stability of bright solitary waves in such fundamental processes as self-action of radiation in media with intensity dependent refractive index and in degenerate three-wave mixing in quadratic nonlinear media [3].

A wide range of parametric processes conserve not only the total energy of the interacting waves but also other energy invariants. These can be, for instance, energies of individual waves or energy unbalance of two waves. Typical examples of such processes are non-degenerate threewave [4] and four-wave mixings [8]. By Noether's theorem every integral of the motion in Hamiltonian models originates from a corresponding symmetry property. Energy invariants are often associated with phase (gauge) symmetries. Although many issues of the dynamics of the multi-wave solitons in models with several symmetries still remain to be understood, their stability threshold in many situations is given by a zero of the determinant of the Jacobi matrix $\left\|\partial_{\kappa_{j}} Q_{i}\right\|$ [2, 6, 7]. Here $Q_{i}$ and $\kappa_{j}$ are respectively the integrals of motion and their associated parameters.

Soliton dynamics in a space where the soliton is localised in some dimensions but extended in one or more others, e.g. consideration of a soliton stripe, raises the problem of modulational instability (MI) along the extra dimensions [1]. MI of solitary waves has been studied in many fields, including plasma physics 漹, fluid dynamics [1, 10] and optics [1, 11, 15]. For instance in optics the above-mentioned extra dimensions might be associated with diffraction and/or group velocity dispersion (GVD). An analytical approach to the low-frequency limit of MI for bright solitary waves was originally developed by Zakharov and Rubenchik [9] for the generalised NLS equation, which has a single phase symmetry. This approach is based on asymptotic expansion near neutrally stable eigenmodes (Goldstone modes) of the solitary wave excitation spectrum. The presence of such neutral modes is directly linked to the symmetries of the model. It follows that systems with a single phase symmetry should be qualitatively similar to the NLS case [9], where the soliton always shows MI in the extra dimension. The unstable mode is of neck type (the soliton stripe breaks into a chain of spots) or snake type (the stripe distorts in zig-zag fashion) depending on the relative signs of the dispersive terms in the localised and extended dimensions. All previous studies of solitary wave MI [1, 10,15] have been restricted to situations with a single phase symmetry, and all do indeed exhibit NLS-like MI. For example, in degenerate three-wave mixing (3WM), which has a single phase symmetry, the first analytical results on MI of solitary waves reported by Kanashov and Rubenchik [11 and recently extended and supported by numerical results 12,13], typically show the neck/snake scenario.

The influence of extra phase symmetries on MI of solitary waves is still an open issue and is the main subject of the present Letter. We will concentrate on nondegenerate $3 \mathrm{WM}$ as a typical and practically important example of a solitonic model with two phase symmetries, motivated by recent theoretical [6,7] and experimental [5,14 advances in the study of quadratic optical solitons.

In this Letter we show that MI of solitary waves in nondegenerate $3 \mathrm{WM}$ reveals a new branch of neck-type instability which can be ascribed to the extra phase symmetry and its corresponding neutral mode. We show that in media with normal GVD this new instability dominates the snake-mode responsible for $\mathrm{MI}$ in the corresponding degenerate 3WM model. Our theoretical approach and the phenomena predicted by it should extend to other solitonic systems of broad interest with similar symmetry properties, e.g. to incoherently coupled NLS equations [16] and non-degenerate 4WM [8].

In non-degenerate $3 \mathrm{WM}$ the evolution of suitably normalised slowly varying field envelopes $E_{m}(m=1,2,3)$ of three waves with carrier frequencies $\omega_{m}\left(\omega_{1}+\omega_{2}=\omega_{3}\right)$ 
propagating in a dispersive and diffractive quadratic nonlinear medium can be modeled [4] by the following system of dimensionless equations:

$$
\begin{aligned}
& i \partial_{z} E_{1}+\alpha_{1} \partial_{x}^{2} E_{1}+\gamma_{1} \partial_{t}^{2} E_{1}+E_{2}^{*} E_{3}=0 \\
& i \partial_{z} E_{2}+\alpha_{2} \partial_{x}^{2} E_{2}+\gamma_{2} \partial_{t}^{2} E_{2}+E_{1}^{*} E_{3}=0 \\
& i \partial_{z} E_{3}+\alpha_{3} \partial_{x}^{2} E_{3}+\gamma_{3} \partial_{t}^{2} E_{3}+E_{1} E_{2}=\beta E_{3}
\end{aligned}
$$

Transverse $x$, longitudinal $z$ and retarded time $t$ coordinates are respectively measured in units of a suitable beam width, diffraction length and GVD parameter. The $\alpha_{m}$ and $\gamma_{m}$ are diffraction and dispersion coefficients referred to these scales, and the wave-vector mismatch is characterised by $\beta$. We neglect spatial and temporal walk-off effects, implicitly assuming that either their spatial and temporal scales are much longer than those associated with diffraction and GVD, or that walkoff is compensated by special techniques, as in recent experiments on temporal solitons in degenerate $3 \mathrm{WM}$ [17]. Henceforth we make the experimentally appropriate choice $\alpha_{1,2}=2 \alpha_{3}=0.5$. We also assume all $\gamma_{m}$ either negative (normal GVD) or positive (anomalous GVD), leaving the case of mixed dispersion for future work. For the sake of simplicity we have restricted our model to one transverse dimension, as in a planar waveguide.

Suppressing the time derivatives for the moment, Eqs. (1) have a family of non-diffracting solitonic solutions $E_{m}=A_{m}(x) e^{i\left(\kappa_{m} z+\phi_{m}\right)}$, where the $A_{m}$ are real, $\kappa_{1,2}=$ $\kappa \pm \delta$ are positive parameters, $\kappa_{3}=2 \kappa>-\beta$ and $\phi_{1,2}=\varphi \pm \psi, \phi_{3}=2 \varphi$, where $\varphi$ and $\psi$ are arbitrary real constants. In general functions $A_{m}(x)$ must be found numerically or approximated variationally [6,7]. The free choice of $\varphi$ and $\psi$ implies two phase (gauge) symmetries, which by Noether's theorem leads to two conserved quantities. These are the total energy $Q=Q_{1}+Q_{2}+2 Q_{3}$, and energy unbalance $Q_{u}=Q_{1}-Q_{2}$, or equivalent combinations of the $Q_{m}=\int d x\left|E_{m}\right|^{2}$. Note that the degenerate case forces $E_{1}=E_{2}$, and thus $\psi=0$, which suppresses one phase symmetry.

Our primary aim here is to study temporal MI due to GVD of these spatial solitons. This is most interesting and important when they are spatially stable. Their stability against purely spatial perturbations has been studied [6,7], yielding a stability boundary which in our notation is given by $\partial_{\delta} Q \partial_{\kappa} Q_{u}=\partial_{\kappa} Q \partial_{\delta} Q_{u}$. Spatially stable domain is in fact almost the entire domain of soliton existence, excluding only a small range of $\kappa, \delta$ values with $\beta<0$ [6, ]1. Close to this region there is also a small domain of bistability [6]. Therefore the existence and nature of temporal MI is the important question for almost all parameter values, and in particular for the entire half-space with $\beta \geq 0$.

To study MI due to GVD we seek solutions of Eqs. (1) in the form of spatial solitons weakly modulated in time at frequency $\Omega \geq 0: E_{m}=\left(A_{m}(x)+\right.$ $\left.\left(V_{m}(x, z)+i W_{m}(x, z)\right) \cos \Omega t\right) e^{i\left(\bar{\kappa}_{m} z+\phi_{m}\right)}$. Setting $V_{m}=$ $v_{m} e^{\lambda z}, W_{m}=w_{m} e^{\lambda z}$, we obtain two eigenvalue problems $\hat{L}_{-} \hat{L}_{+} \vec{v}=\lambda^{2} \vec{v}$ and $\hat{L}_{+} \hat{L}_{-} \vec{w}=\lambda^{2} \vec{w}$, where $\vec{v}=$ $\left(v_{1}, v_{2}, v_{3}\right)^{T}, \vec{w}=\left(w_{1}, w_{2}, w_{3}\right)^{T}$ and

$$
\hat{L}_{ \pm}=\left[\begin{array}{ccc} 
\pm \hat{L}_{1} & A_{3} & \pm A_{2} \\
A_{3} & \pm \hat{L}_{2} & \pm A_{1} \\
\pm A_{2} & \pm A_{1} & \pm \hat{L}_{3}
\end{array}\right]
$$

$\hat{L}_{m}=\alpha_{m} \partial_{x}^{2}-\gamma_{m} \Omega^{2}-\xi_{m}$, where $\xi_{1,2}=\kappa \pm \delta$ and $\xi_{3}=$ $2 \kappa+\beta$. Note that $\hat{L}_{ \pm}$are self-adjoint, so $\hat{L}_{-} \hat{L}_{+}$and $\hat{L}_{+} \hat{L}_{-}$are adjoint operators with identical spectra. It is thus enough to consider the spectrum of one of these operators, e.g. $\hat{L}_{+} \hat{L}_{-}$. We are particularly interested in the discrete spectrum, with eigenfunctions exponentially decaying at $x \rightarrow \pm \infty$.

In general the stability problem can only be solved numerically, but for small absolute values of $\lambda$ we can obtain some analytical results. Our two phase symmetries, plus the Galilean one, generate three neutrally stable $(\lambda=0)$ eigenmodes of $\hat{L}_{+} \hat{L}_{-}$at $\Omega=0$. These are: $\vec{w}_{\varphi}=\left(A_{1}, A_{2}, 2 A_{3}\right)^{T}, \vec{w}_{\psi}=\left(A_{1},-A_{2}, 0\right)^{T}, \vec{w}_{x}=$ $\left(x A_{1}, x A_{2}, 2 x A_{3}\right)^{T}$. Assuming that $\Omega \ll 1$ we can express $\vec{w}$ as a linear combination of $\vec{w}_{\varphi}, \vec{w}_{\psi}, \vec{w}_{x}$ and then use an asymptotic approach to find eigenvectors and corresponding eigenvalues. This approach can be applied only if all other eigenmodes have eigenvalues obeying the condition $|\lambda|>\Omega$. In practice this only excludes a small neighborhood of the spatial stability boundary discussed above.

Three eigenvalue pairs $\pm \lambda$ are obtained from solvability conditions of the first order problems. One, associated with the asymmetric eigenvector $\vec{w}_{x}$, obeys

$$
\lambda_{x}^{2} \simeq-\frac{2 \Omega^{2}}{Q} \int d x \sum_{m=1}^{3} \gamma_{m}\left(\partial_{x} A_{m}\right)^{2}
$$

Clearly the asymmetric mode is unstable for normal GVD, which corresponds to the snake instabilities found in NLS [9,10] and degenerate 3WM [11,12] models.

The other two eigenvalue pairs are associated with linear combinations of the spatially symmetric vectors $C_{\varphi} \vec{w}_{\varphi}+C_{\psi} \vec{w}_{\psi}$, and thus with neck-type instabilities. They are the roots of

$$
a \lambda^{4}+b \Omega^{2} \lambda^{2}+c \Omega^{4}=0
$$

where $a=\left(\partial_{\delta} Q \partial_{\kappa} Q_{u}-\partial_{\kappa} Q \partial_{\delta} Q_{u}\right) / 2, b=\partial_{\kappa} Q\left(\gamma_{1} Q_{1}+\right.$ $\left.\gamma_{2} Q_{2}\right)+\partial_{\delta} Q_{u}\left(\gamma_{1} Q_{1}+\gamma_{2} Q_{2}+4 \gamma_{3} Q_{3}\right)+\left(\partial_{\kappa} Q_{u}+\right.$ $\left.\partial_{\delta} Q\right)\left(\gamma_{2} Q_{2}-\gamma_{1} Q_{1}\right)$, and $c=-8\left(\gamma_{1} \gamma_{2} Q_{1} Q_{2}+\gamma_{2} \gamma_{3} Q_{2} Q_{3}+\right.$ $\left.\gamma_{1} \gamma_{3} Q_{1} Q_{3}\right)$. These expressions are quite complicated, but yield some important general results. Clearly $c$ is negative when all $\gamma_{m}$ of the same sign. Since $a>0$ throughout the spatially monostable domain, it follows that the two roots $\lambda^{2}$ are always real and of opposite sign, so that there is always an unstable neck-type mode. Thus we establish coexistence and competition of neck and snake instabilities for normal GVD. This is a novel feature of the present model, quite different from previous analytical results for NLS [9,10] and degenerate 3WM [11, 12], 
where the snake instability is the only one for normal GVD. This is because only one symmetric neutral mode exists in models with a single phase symmetry, and it generates instability only for anomalous GVD. In nondegenerate $3 \mathrm{WM}$ there are two neck modes, one stable for normal GVD and unstable for anomalous GVD, and vice versa, since $b$ is odd in the $\gamma_{m}$.

Simple analytic expressions for growth rates of these neck modes can be obtained in several special cases, e.g. in the case of second harmonic generation $\left(\omega_{1}=\omega_{2}\right)$. Setting $\gamma_{1}=\gamma_{2}$ and $\delta=0$ (readily achieved in experiment [5]) the two eigenmodes have either $C_{\varphi}=0$ or $C_{\psi}=0$, with eigenvalues

$$
\lambda_{\psi}^{2} \simeq 2 \gamma_{1} \Omega^{2} \frac{Q_{1}}{\partial_{\delta} Q_{1}}, \quad \lambda_{\varphi}^{2} \simeq \frac{4 \Omega^{2}}{\partial_{\kappa} Q}\left(\gamma_{1} Q_{1}+2 \gamma_{3} Q_{3}\right) .
$$

For $\delta=0, \partial_{\kappa} Q$ is positive and $\partial_{\delta} Q_{1}$ is negative in the spatially monostable domain. Thus the novel neck instability for $\gamma_{1}<0$ can be directly attributed to the gauge symmetry in the differential phase $\psi$ and its associated neutral mode $\vec{w}_{\psi}$. On the other hand $\lambda_{\varphi}$ is associated with the usual neck MI for anomalous GVD in models with a single gauge symmetry 99 13]. The expression for $\lambda_{\psi}^{2}$ holds also (when $Q_{u}=0$ ) for other solitonic models with a differential phase symmetry. Note, however, that $\partial_{\delta} Q_{1}$ can generally have either sign, leading to instability with either normal or anomalous GVD.

Solving the eigenvalue problem numerically, we find that in low-frequency limit the instability growth rates precisely match those predicted by our perturbation theory, see Fig. 1(a), (b). As $\Omega$ is increased each MI gain curve reaches a maximum and then decreases. A typical example of the maximal MI growth rate vs $\beta$ is presented in Fig. 1(c). Similar plots for $Q_{u} \neq 0$ and across wide range of $\gamma_{m}$ values show the same behaviour [18]. Thus we conclude that for normal GVD the new neck instability strongly dominates the snake one. Note that its growth rate is maximised, as Fig. 1(a), (b) illustrate, for $Q_{u}=0$. For normal GVD the unstable eigenfunctions become weakly confined and develop oscillating tails as $\Omega$ increases. Because this increases computer demand, we have plotted growth rates in Fig. 1(a) only for $\Omega$ values corresponding to well-localised eigenmodes. Physically, broader eigenmodes have weaker overlap with the soliton, and hence lesser gain.

Spatial profiles of the symmetric eigenfunctions at maximum gain $\left(\Omega=\Omega_{\max }\right)$ are presented in Fig. 2 . Despite this being well beyond the perturbative limit in which expressions (4) apply, the novel neck MI eigenmode still has qualitatively the same form as $\vec{w}_{\psi}$, i.e. $w_{1}=-w_{2}, w_{3}=0$, indicating that the $\psi$ phase symmetry underlies the instability through the whole range of $\Omega$. Similarly, the ustable neck mode for anomalous GVD is evidently associated with the $\varphi$ symmetry.

To test our linear stability analysis and study the nonlinear evolution we performed an extensive series of computer simulations of the system (1) with initial conditions in form of a soliton stripe perturbed by spatio-temporal white noise of order $1 \%$. Typical simulation results are presented in Fig. 3 and they fully support our predictions. We chose the size of the computational window in the time domain to be $18 \pi / \Omega_{\max }$, and the initial soliton stripe rapidly develops nine humps, in accord with the stability analysis. During further evolution the modulated stripe forms into a train of pulses which either spread (normal GVD) or form a persistent chain of threewave optical bullets (anomalous GVD). Due to the initial noise, modes from a band of frequencies close to $\Omega_{\max }$ are able to grow and compete, and hence the modulations in Fig. 3 are somewhat irregular.

A striking difference between Figs. 3(a,c) is that the initially imposed translational symmetry of the solitary stripe along the time dimension is broken in different ways. For normal GVD interleaved intensity peaks of $E_{1}$ and $E_{2}$ are formed, while for anomalous GVD the intensity peaks coincide. (Each amplitude is modulated with period $\simeq 2 \pi / \Omega_{\max }$.)

This difference is directly related with the spatial form of the most unstable eigenvectors. In the case of normal GVD $w_{1}$ and $w_{2}$ are out of phase and $w_{3}=0$, see Fig. 2(a), leading to the interleaving. Since $E_{1} E_{2}$ drives $E_{3}$, the intensity profile of the second harmonic becomes modulated with period $\pi / \Omega_{\max }$, see Fig. $3\left(\mathrm{a}_{3}\right)$. Because the overlap of the three fields is diminished by this evolution, mutual trapping becomes impossible and the whole structure eventually spreads through diffraction and dispersion, see Fig. 3(b). For anomalous GVD, all three components of most unstable eigenvector are in phase, see Fig. 2(b), and thus all three intensities become modulated with the same temporal period, see Fig. $3\left(c_{1}\right),\left(c_{2}\right),\left(c_{3}\right)$. This provides conditions for mutual self-trapping of the filaments, see Fig. 3(d).

The predicted instabilities can be experimentally observed in a nonlinear material longer than the MI gain length $l_{g} \sim \lambda^{-1}$ and with pulse width order of several $2 \pi / \Omega_{\max }$ or more. Following Ref. [12], typical values for $\mathrm{KTP} l_{g} \sim 1 \mathrm{~cm}$ and $2 \pi / \Omega_{\max } \sim 10^{-12} \mathrm{~s}$. Fig. $1(\mathrm{c})$ shows that the new neck instability has the highest gain and is thus most easily observable. Artificially birefrigent semiconductor materials, which are highly nonlinear, seem quite promising, based on a recent experiments on $3 \mathrm{WM}$ [19]. Waveguides containing Bragg structures might be very suitable for observation of the MI phenomena predicted here because of their large and controllable dispersion 20.

In summary, we have analysed and described dispersive MI of spatial solitons due to non-degenerate 3WM. Using this model as a typical example we generalised a previous analytical approach to MI [9] to models with two phase symmetries. We found that the extra neutral mode associated with the additional phase symmetry gives rise to a new branch of MI. This is symmetric (of neck type), and is found to dominate the asymmetric (snake) instability which is the only MI for normal GVD in systems possessing just one phase symmetry. This result enables a new 
understanding of the dynamics of multi-component solitary waves in terms of their phase symmetry properties. The MI phenomena which we have described for $3 \mathrm{WM}$ are likely to be generic in other solitonic and nonlinear wave models with two phase symmetries.

This work was partially supported by EPSRC grant GR/L 27916.

[1] E. Kuznetsov, A. Rubenchik, and V. Zakharov, Phys. Rep. 142, 103 (1986).

[2] V.G. Makhankov, Y.P. Rybakov, and V.I. Sanyuk, Physics-Uspekhi 37, 113 (1994).

[3] M.G. Vakhitov and A.A. Kolokolov, Sov. Radiophys. 16, 783 (1973); D.E. Pelinovsky, A.V. Buryak, and Y.A. Kivshar, Phys. Rev. Lett. 75, 591 (1995).

[4] Y.N. Karamzin and A.P. Sukhorukov, Sov. Phys. JETP 41, 414 (1976).

[5] W.E. Torruelas et al., Phys. Rev. Lett. 74, 5036 (1995); R.A. Fuerst et al., Opt. Lett. 22, 1748 (1997).

[6] A.V. Buryak, Y.S. Kivshar, and S. Trillo, Phys. Rev. Lett. 77, 5212 (1997); J. Opt. Soc. Am. B 14, 3110 (1997).

[7] U. Peschel et al., Phys. Rev. E 55, 7704 (1997); D. Mihalache et al., Phys. Rev. E 56, R6249 (1997).

[8] P.B. Lundquist, D.R. Andersen, and Y.S. Kivshar, Phys. Rev. E, 57, 3551 (1998) and refs. therein.

[9] V.E. Zakharov and A.M. Rubenchik, Sov. Phys. JETP 38, 494 (1974).

[10] B.I. Cohen, K.M. Watson, and B.J. West, Phys. Fluids 19, 345 (1976); P.A.E.M. Janssen and J.J. Rasmussen, Phys. Fluids 26, 1279 (1983).

[11] A.A. Kanashov and A.M. Rubenchik, Physica D 4, 122 (1981).

[12] A. De Rossi et al., Phys. Rev. E 56, R4959 (1997).

[13] D.V. Skryabin and W.J. Firth, Opt. Commun. 148, 79 (1998); D.-M. Baboiu and G.I. Stegeman, Opt. Lett. 23, 31 (1998).

[14] R.A. Fuerst et al., Phys. Rev. Lett. 78, 2756 (1997).

[15] A.V. Mamaev et al., Phys. Rev. A 54, 870 (1996).

[16] A.L. Berkhoer and V.E. Zakharov, Sov. Phys. JETP 31, 486 (1970).

[17] P. Di Trapani et al., Phys. Rev. Lett. 81, 570 (1998).

[18] The expressions for $\lambda_{x}^{2}$ and $\lambda_{\psi}^{2}$ show that if $-\gamma_{3}$ is large enough the snake MI has the higher gain. However these expressions are only valid in the low-frequency limit, and numerics show that for $\Omega=\Omega_{\max }$ the neck MI dominates the snake even more strongly than in the examples shown in Fig. 1.

[19] A. Fiore et al., Nature 391, 463 (1998).

[20] C. Conti, S. Trillo, and G. Assanto, Phys. Rev. Lett. 78, 2341 (1997); H. He and P.D. Drummond, ibid., 4311 (1997).
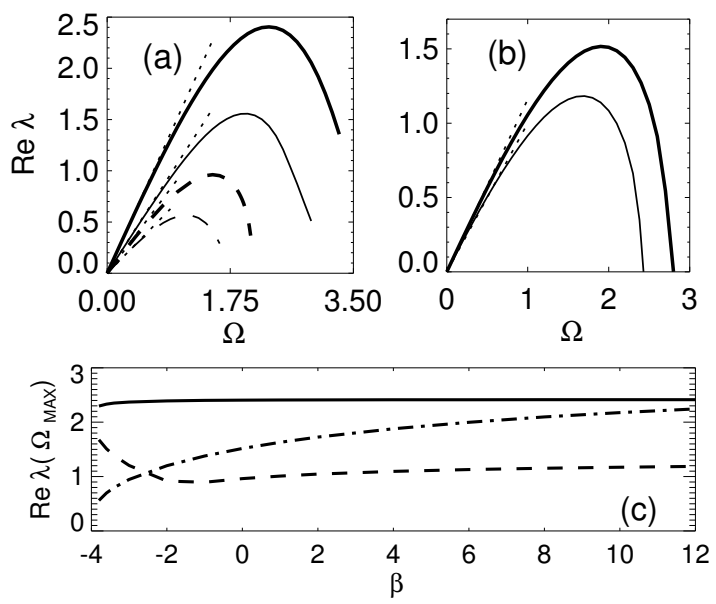

FIG. 1. (a-b) Instability growth rate vs $\Omega: Q=65, \beta=0$. Thick (thin) lines are for $\kappa=2, \delta=0, Q_{u}=0(\kappa=2.075$, $\delta=1.525, Q_{u}=-36$ ). Full (dashed) lines correspond to neck (snake) MI. Dotted lines are perturbative results. (a) Normal dispersion: $\gamma_{1,2}=2 \gamma_{3}=-0.5$. (b) Anomalous dispersion: $\gamma_{1,2}=2 \gamma_{3}=0.5$. (c) MI growth rate at $\Omega=\Omega_{\max }$ vs $\beta$ for $\kappa=2, \delta=0$. Full (dashed) lines correspond to neck (snake) MI for $\gamma_{1,2}=2 \gamma_{3}=-0.5$; dot-dashed line to neck MI for $\gamma_{1,2}=2 \gamma_{3}=0.5$
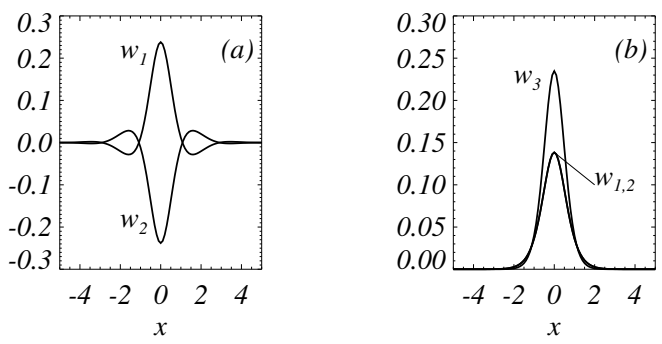

FIG. 2. Most unstable eigenmodes $\left(\Omega=\Omega_{\max }\right)$ for $\beta=\delta=0, \kappa=2$. (a) Normal dispersion: $\gamma_{1,2}=2 \gamma_{3}=-0.5$; (b) Anomalous dispersion: $\gamma_{1,2}=2 \gamma_{3}=0.5$. 

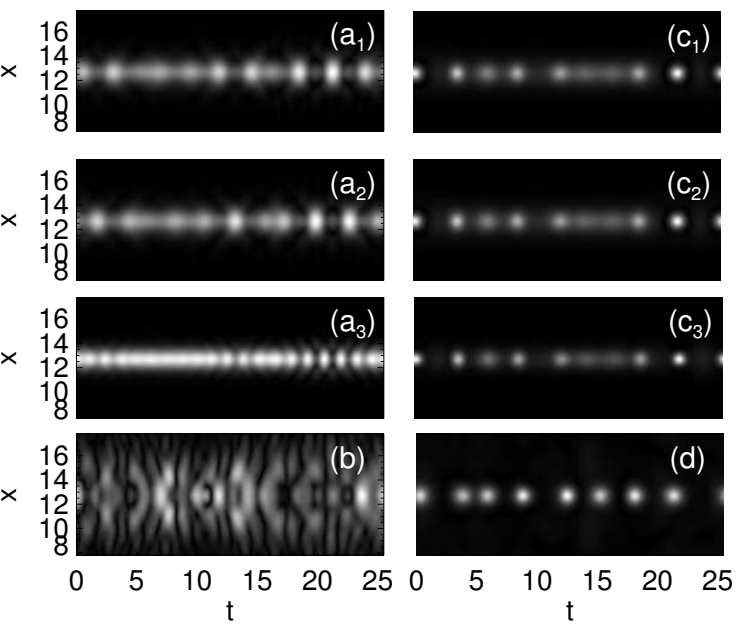

FIG. 3. Development of noise-induced instability of spatial soliton stripe: $\kappa=2, \delta=\beta=0$. Left (right) panels for $\gamma_{1,2}=2 \gamma_{3}=-0.5\left(\gamma_{1,2}=2 \gamma_{3}=0.5\right) .\left(\mathrm{a}_{m}\right)\left|E_{m}\right|$ at $z=2.7$, (b) $\left|E_{1}\right|$ at $z=4.5,\left(\mathrm{c}_{m}\right)\left|E_{m}\right|$ at $z=5.4$, (d) $\left|E_{1}\right|$ at $z=10.8$. 